\title{
A Review on the Research Progress of Inducible Nitric Oxide Synthase in the Pathogenesis of Pancreatic Malignancy
}

\author{
Huimin Xiong1, Ruiyao Wang2*, Chao Zhang3 \\ ${ }^{1}$ Medical College of Ankang University, Ankang City, Shaanxi Province, China \\ ${ }^{2} X i$ 'an Hospital of Traditional Chinese Medicine for Encephalopathy of Shaanxi Province, Xi'an City, Shaanxi Province, \\ China \\ ${ }^{3}$ Emergency Department, Dali County Hospital of Traditional Chinese Medicine, China \\ *Corresponding author: Ruiyao Wang, 1947843671@qq.com

\begin{abstract}
Pancreatic cancer is a common tumor of the digestive system, at present, the pathogenesis is still unclear, but in the current research on the pathogenesis of pancreatic malignant tumors, the research on inducible nitric oxide synthase is particularly extensive. Therefore, this article focuses on the research progress of inducible nitric oxide synthase in the pathogenesis of pancreatic cancer. This is a review.
\end{abstract}

Keywords: Inducible nitric oxide synthase; Pancreatic cancer; Research progress

Publication date: July 2021; Online publication: July 31, 2021

\section{Introduction}

Pancreatic cancer is a highly malignant tumor of the digestive system. In recent years, the incidence and mortality of pancreatic cancer have continued to rise. Epidemiological data show that pancreatic cancer is expected to become the second leading cause of cancer and related deaths by $2030^{[1]}$. Pancreatic ductal adenocarcinoma (PDAC) is the most common type of pancreatic cancer, accounting for more than $90 \%$ of pancreatic cancer. PDAC has a highly inflammatory tumor microenvironment. Recent studies have shown that inflammatory mediators produced by tumors and mesenchymal cells are involved in the occurrence and development of PDAC ${ }^{[2]}$. Nitric oxide (NO) is a free radical and an important mediator of immune and inflammatory response. In addition to playing a role in key biological processes such as vasodilation and neurotransmission, it is also an important immune and inflammatory response mediator. However, there is a large amount of evidence that NO plays an important role in the occurrence and development of cancer [3-8].

NO is produced by the family of nitric oxide synthase, including endothelial nitric oxide synthase (eNOS) and neuronal nitric oxide synthase (nNOS) and induced expression after injury type nitric oxide synthase (iNOS). eNOS and nNOS belong to the constitutive isomer NOS and only produce a small amount of NO, while iNOS is the inducible isomer NOS, which will produce a large amount of NO under the action of external factors ${ }^{[3]}$. A large number of clinical studies of PDAC ${ }^{[9-14]}$ show that the massive production of NO and the expression of iNOS in the course of PDAC are closely related to PDAC, which can promote the occurrence of cancer and angiogenesis ${ }^{[15-17]}$.

Jiangwei Liu ${ }^{[18]}$ tested the expression level of iNOS in patients with pancreatic ductal carcinoma and found that the protein expression rate of iNOS was $62.7 \%$, which was related to lymph node metastasis. In 
addition, their group also found that the expression of iNOS was closely related to the expression of COX 2. iNOS and COX 2 may play a synergistic role in the occurrence and development of pancreatic cancer, and promote tumor angiogenesis and metastasis.

In order to study the role of iNOS in PDAC in depth, Wang ${ }^{[19]}$ established a PDAC genetically engineered mouse model (KPC mouse) and an iNOS knock-out (iNOS-/-) KPC mouse model (NKPC mouse), and found that the expression of iNOS in KPC mice was significantly increased and the survival cycle was significantly shortened, while the progression of PDAC in NKPC mice was slowed down and the survival cycle was significantly prolonged. Their research group further separated KPC and NKPC mouse primary tumor cells, and used the Xcelligence system to monitor the proliferation of tumor cells in real time and dynamically. It was found that the proliferation of primary tumor cells in NKPC mice was reduced, and the apoptosis index Caspase-3 was detected. Compared with KPC mice, the apoptosis of pancreatic tumor cells in NKPC mice was significantly increased. In addition, qRT-PCR found that the expression of E-cadherin, a phenotypic marker of EMT, was significantly higher in primary tumor cells of NKPC mice than in KPC mice. Tip: iNOS is involved in the progression of PDAC, and iNOS gene knockout may delay the progression of PDAC ${ }^{[20-21]}$.

In summary, iNOS is involved in the occurrence of PDAC, and the in-depth study of the role of iNOS in PDAC will provide new ideas for the clinical treatment of PDAC.

\section{Disclosure statement}

The author declares no conflict of interest.

\section{References}

[1] Rahib L, Smith BD, Aizenberg R, 2014, Projecting Cancer Incidence and Deaths to 2030: The Unexpected Burden of Thyroid, Liver, and Pancreas Cancers in the United States. Cancer Res. 74:2913-2921.

[2] Gukovsky I, Li N, Todoric J, 2013, Inflammation, Autophagy, and Obesity: Common Features in the Pathogenesis of Pancreatitis and Pancreatic Cancer. Gastroenterology. 144:1199-1209 e1194.

[3] Tamir S, Gal A, Tannenbaum SR, 1995, Nitric Oxide Production in Relation to Spontaneous Bcell Lymphoma and Myositis in SJL Mice. Cancer Res. 55:4391-4397.

[4] Tamir S, Tannenbaum SR, 1996, The Role of Nitric Oxide (NO.) in the Carcinogenic Process. Biochim Biophys Acta. 1288:F31-36.

[5] Jenkins DC, Charles IG, 1995, Roles of Nitric Oxide in Tumor Growth. Proc Natl Acad Sci U S A. 92:4392-4396.

[6] Hussain SP, Hofseth LJ, Harris CC, 2003, Radical Causes of Cancer. Nat Rev Cancer. 3:276-285.

[7] Lim KH, Ancrile BB, 2008, Tumour Maintenance is Mediated by eNOS. Nature. 452:646-649.

[8] Lampson BL, Kendall SD, 2012, Ancrile BB. Oncotarget 53003, Targeting eNOS in Pancreatic Cancer. Cancer Res.

[9] Vickers SM, Green M, 1999, Association of Increased Immunostaining for Inducible Nitric Oxide Synthase and Nitrotyrosine with Fibroblast Growth Factor Transformation in Pancreatic Cancer. Arch Surg. 134:245-251.

[10] Chung HT, 2001, Nitric Oxide as a Bioregulatorof Apoptosis. Biochem Biophys Res Commun. 282:1075-1079.

[11] Li A, Morton JP, et al., 2014, Fascin is Regulated by Slug, Promotes Progression of Pancreatic 
Cancer in Mice, and is Associated with Patient Outcomes. Gastroenterology. 146:1386-1396 e1381-1317.

[12] Hou YC, Chao YJ, Tung HL, et al., 2014, Co-expression of CD44-Positive/CD133-Positive Cancer Stem Cells and CD204-Positive Tumor-Associated Macrophages is a Predictor of Survival in Pancreatic Ductal Adenocarcinoma. Cancer. 120:2766-2777.

[13] Weizman N, Krelin Y, 2014, Macrophages Mediate Gemcitabine Resistance of Pancreatic Adenocarcinoma by Upregulating Cytidine Deaminase. Oncogene. 33:3812-3819.

[14] Sanford DE, Belt BA, et al., 2013, Inflammatory Monocyte Mobilization Decreases Patient Survival in Pancreatic Cancer: A Role for Targeting the CCL2/CCR2 Axis. Clin Cancer Res. 19: 3404-3415.

[15] Schetter AJ, Heegaard NH, Harris CC, 2010, Inflammation and Cancer: Interweaving Microrna, Free Radical, Cytokine and p53 Pathways. Carcinogenesis. 31:37-49.

[16] Okayama H, Saito M, 2013, NOS2 Enhances KRAS-Induced Lung Carcinogenesis, Inflammation and MicroRNA-21 Expression. Int J Cancer. 132: 9-18.

[17] Dillhoff M, Liu J, 2008, MicroRNA-21 is Overexpressed in Pancreatic Cancer and a Potential Predictor of Survival. J Gastrointest Surg. 12: 2171-2176.

[18] Liu J, Li K, Dou K, 2003, The Expression of iNOS and COX-2 in Pancreatic Cancer and its Clinical Significance. Cancer Research on Prevention and Treatment, 30(5): 361-361

[19] Wang J, He PJ, et al., 2016, Inducible Nitric Oxide Synthase Enhances Disease Aggressiveness in Pancreatic Cancer. Oncotarget, 7: 52993-53004.

[20] Paik JH, Kollipara R, Tothova Z, et al., 2007, FoxOs are Lineage-Restricted Redundant Tumor Suppressors and Regulate Endothelial Cell Homeostasis. Cell. 128:309-323.

[21] Tothova Z, Kollipara R, et al., 2007, FoxOs are Critical Mediators of Hematopoietic Stem Cell Resistance to Physiologic Oxidative Stress. Cell. 128:325-330. 